\title{
U.K. Stock Market Inefficiencies and the Risk Premium
}

\author{
Antonis Demos \\ Athens University of Economics and Business, Greece \\ George Vasillelis \\ Imperial College and Dresdner-Kleinwort-Benson Bank, U.K.
}

The stock market predictability has been a favorite topic of scholars and practitioners alike. It seems that some small predictability is present in all major stock markets worldwide. This predictability can be attributed to the risk premium structure and/or to inefficiencies present in the markets. This paper investigates the predictability of returns of some major shares listed in the London Stock exchange, using economic as well as accounting variables. We first measure the predictability of these variables by regressing individual stock returns on their corresponding accounting variables and the economic ones. Second, we estimate for the returns a seasonal latent factor model with time varying volatility. Provided that our measure of risk is an adequate one, the residuals of this estimation are free of the predictability of risk premium, and consequently one expects that any accounting and factor economic variables would have no predictive power. An $L M$-type test is developed and employed to indicate that indeed the U.K. stock market predictability is due to the risk premium structure, and the explanatory power of the variables considered here is due to them being an approximation of risk. However, when we perform the test jointly for all assets, we reject the zero predictability hypothesis at $5 \%$ but not at $1 \%$ (JEL: G12, G14, C10).

Keywords: conditional heteroskedastic latent factor, LM test, stock returns.

\footnotetext{
* We are grateful to Stelios Arvanitis, Ritsa Panagiotou, Apostoli Philippopoulo, Helen Stavropoulou, Thanasi Stengo, and especially Enrique Sentana. We would like to thank the participants at the $4^{\text {th }}$ Annual Conference of the Multinational Finance Society (1997) and especially our discussant Chris Adcock, Econometric Society European Meeting (1998), the seminar participants at Reading University and Athens University of Economics and Business. Our special thanks to two anonymous referees for their constructive comments. All remaining errors are ours.
}

(Multinational Finance Journal, 2007, vol. 11, no. 1/2, pp. 97-122)

(C) by Multinational Finance Society, a nonprofit corporation. All rights reserved. DOI: $10.17578 / 11-1 / 2-4$ 


\section{Introduction}

Since the end of the seventies there has been growing evidence that macroeconomic and accounting variables as well as seasonal regularities can predict stock returns. Some authors interpret this as a rejection of the Semistrong-Form Efficient Market Hypothesis (EMH) (see, e.g., Fama [1991]). Others, however, argue that tests which reject the above hypothesis fail to model risk appropriately. Two main methods have been adapted to model conditional risk premia. The first is to accept a priory that any predictability is due to the fact that the time variation of these variables approximate risk premia. Alternatively, in a multivariate set-up, the latent factor model of Diebold and Nerlove (1989) and the factor GARCH of Engle (1987) are the best-known examples of how to obtain a parsimonious representation of risk premia as functions of the conditional second moments of returns.

This paper develops a procedure to investigate stock return predictability in a time series setup and applies this procedure to individual stocks quoted at the London Stock Exchange. This involves first examining whether there is any predictability from a large variety of accounting and economic variables. To account explicitly for risk premia time variation we estimate a multivariate conditional Arbitrage Pricing Theory (APT) model. This way ensures that first, the predictable component in returns is a risk premium, and second, by having a seasonal factor that is zero in non-January months, the January effect is due to a higher risk premium for this month. Provided that the asset pricing model is correctly specified and the $E M H$ holds, the predictability of any lagged economic and/or accounting variables should be statistically insignificant. To test this we develop a robust $L M$-type test. If the returns' predictability is due to the risk premium, the explanatory power of the limiting factors representing portfolios and the instruments on the conditional APT residuals must be insignificant. Alternatively, if the $L M$ test rejects the null hypothesis of zero additional predictability, then either the asset pricing model is not well-specified, and/or the predictability of the instruments is due to inefficiencies in the market.

We apply the developed procedure to monthly excess returns of individual companies listed in the 100 FT Index. We document the predictability of the accounting and economic variables for the individual stock returns in the U.K. market. In terms of the conditional $A P T$ model we confirm that the risk-return relationship is different in 
January compared to other months, however this relationship is different as compared with the U.S. experience. Finally, in terms of U.K. market efficiency, our results strongly indicate that the predictability of the accounting and economic variables considered here, is due to risk premium and disappears as soon as its structure is taken into account.

In section II we provide a general analysis of the $E M H$ topic and analyze the potentially predicting instruments. In section III we present the conditional APT model and develop the $L M$ test along with a Monte Carlo exercise to assess its properties in section IV. Section V presents the empirical results. We conclude in section VI.

\section{Instruments and the Information Set}

The most commonly used model of stock returns makes the expected excess return a function of volatility (see, e.g., Merton [1980]). If expectations are also assumed to be rational, then we have the $E M H$ model with the implication that information available at time $t-1$ cannot help us predict risk-adjusted excess returns. An important alternative to the $E M H$ model are the so called "fads" or "noise traders" models (see, e.g., Shiller [1984], Sentana and Wadhwani [1991], and Campbell and Kyle [1993]). Consequently, the predictability of risk-adjusted returns may be interpreted as evidence in favor of the "fads" model and against the specific parameterization of the $E M H$ model. Expected and/or unexpected macro variables as well as various default or term spreads, interest rates, stock and/or bond indices, and stock index dividend yields are very popular economic indicators that are employed to predict stock returns. ${ }^{1}$ However, this predictability could be attributed to either the structure of risk premia or to market inefficiencies.

Our lagged economic information set includes, among other variables, the retail price index, the M0 money supply and industrial production. From the price index we construct the inflation series and we estimate, recursively, the expected inflation using the procedure developed in Ferson and Harvey (1991). The same procedure is applied to the M0 and industrial production data. We evaluated the term spread

1. See, e.g., Campbell (1987), Fama (1981), Fama and Schwert (1977), Harvey (1991), Lee (1997), Lo and MacKinlay (1997), Poon and Taylor (1991), Sentana and Wadhwani (1991), and Wong et al. (2001). 
as the difference of the 20-year and 1-month rates. As measures of interest rate trends we use the monthly changes of the two Treasury Bills and the Government Bond. Hence, our set of economic instruments consists of 26 variables (see appendix, table A).

Out of these 26 instruments we choose 16 canonical factors, which explain $86.6 \%$ of the maximum correlation between these instruments and the excess returns under consideration. This approach is most appropriate in our case, as the objective is to form factors that are maximally correlated with the returns under consideration. ${ }^{2}$ It is also closely related to the Lo and MacKinlay (1997) Maximally Predictable Portfolio $(M P P)$ one. Furthermore, this procedure was chosen under power considerations for the application of the $L M$ test (see section V). Moreover, our set includes variables that are considered to be stationary as well as nonstationary. ${ }^{3}$ Applying the canonical correlation procedure ensures that the linear combinations of these instruments will be correlated with linear combinations of stock returns. Finally, our approach is more general than just putting the stationary instruments as predictors, as in our case we also use any stationary linear combination of the nonstationary processes.

Turning our attention now to the accounting variables, nine are included in our accounting information set (see appendix, table B). ${ }^{4}$ Another stylized fact of monthly stock market returns is the January Effect (documented internationally see, e.g., Gultekin and Gultekin [1983]). Hence, a January dummy is included in our information set, to take into account the tendency of stock returns to be higher in January than the rest of the year. Consequently, we run the following regression for each of the 45 common stock excess returns:

$$
r_{i t}=\gamma_{0}+\gamma_{1} D_{t}+\gamma_{2} x_{i 1 t-1}+\ldots+\gamma_{9} x_{i 8 t-1}+\gamma_{10} f_{1 t}^{c}+\ldots \gamma_{25} f_{16 t}^{c}+\eta_{i t}
$$

where $D_{t}$ is a January dummy, $r_{i t}$ is the excess return—over and above

2. This method is also supported theoretically as a method of forming portfolios that are maximally correlated with factor scores (see Huberman et al. [1987]).

3. This nonstationarity makes the methods of principal component and factor analysis inappropriate.

4. See, e.g., Al-Qenae et al. (2002), Campbell and Shiller (1998), Chan et al. (1991), Fama and French (1988) and (1996), Jermakowicz and Gornik (1998), Shiller (1984), and Wong et al. (2001). 
the safe rate-of the $i^{\text {th }}$ company, $x_{i 1 t-1}$ to $x_{i 8 t-1}$ are the 8 accounting variables of the same company, and $f_{1 t}^{c}$ to $f_{16 t}^{c}$ are the 16 canonical factors. Hence, there are 25 predictive variables, 26 including the constant $\gamma_{0}{ }^{5}$

\section{A Time-Varying Volatility Seasonal Latent Factor Model}

From the above section it is clear that the key of this controversy is the modeling of the risk premium, and specifically, its time variation. Consequently, an unconditional setting may not be appropriate, since most recent asset pricing models call for conditional moments (see Keim and Stambaugh [1986]). The theoretical asset pricing model is based on King et al. (1994), where more details can be found. The model is based on a universe with a countably infinite number of primitive assets. The gross return of asset $i$ in period $t, R_{i t}(i=1,2, \ldots)$, is generally uncertain since the asset is risky. However, we assume the existence of a safe asset, whose return, $R_{0 t}$, is determined at the end of period $t-1$, and consequently is known to agents. The basic assumption made on the stochastic structure of asset returns is that the unanticipated component of asset $i$ 's return has a conditional factor representation, where the common factors capture systematic risk affecting all assets. We assume (without loss of generality) that they are conditionally orthogonal and that they have time varying conditional variances, $\lambda_{j t}(j$ $=1,2, \ldots, k)$. For the idiosyncratic terms, we assume that they are conditionally uncorrelated to each other, which corresponds to an exact conditional $k$ factor structure (see Chamberlain and Rothschild [1983]), and have constant conditional variances $\omega_{i}$.

Let $R_{f 1}, \ldots, R_{f k t}$ be the returns on $k$ limiting factor representing portfolios, i.e., unit cost well-diversified portfolios of risky assets which have unit loading on only one factor and zero loadings on the rest $k-1$. Then, in this framework, it is possible to prove that the excess return, $r_{i t}$ $=R_{i t}-R_{0 t}$, of the $i^{\text {th }}$ asset is:

$$
\begin{gathered}
r_{i t}=\beta_{i 1 t} \lambda_{1 t} \tau_{1 t}+\ldots+\beta_{i k t} \lambda_{k t} \tau_{k t}+\beta_{i 1 t} f_{1 t}+\ldots+\beta_{i k t} f_{k t}+\in_{i t} \\
(i=1,2, \ldots)
\end{gathered}
$$

5. Notice that the above regressions are sensitive to the Stambaugh (1999) criticism for small-sample bias. Nevertheless, in our application this bias is of small order as we employ monthly data (see Ang and Bekaert [2001]). 
where $\tau_{i t}$ is the price of risk of the $i^{\text {th }}$ factor $(i=1,2, \ldots, k)$.

In this paper we consider a two-factor model for the $N$ individual stocks. The first one has a time varying conditional variance given by:

$$
\lambda_{1 t}=\psi_{0}+\psi_{1} E_{t-1}\left(f_{1 t-1}\right)+\psi_{2} E_{t-1}\left(f_{1 t-1}^{2}\right)+\psi_{3} \lambda_{1 t-1}
$$

so that $\lambda_{1 t}$ is a measurable function of the information set and consequently a correction term is included, which reflects the uncertainty related to the estimation of this unobserved factor (see Harvey et al. [1992]). Furthermore, $E_{t-1}\left(f_{1 t-1}\right)$ incorporates the so-called leverage effect, i.e., a decline in share prices increases volatility by more than a price increase of the same size (see Campbell and Hentschel [1992] for U.S. and Demos et al. [1994] for the U.K.). Hence, for $\lambda_{1 t}$ the QGARCH $(1,1)$ model of Sentana (1995), is employed.

Following Demos et al. (1994), the second factor is a seasonal one, which captures the January effect, with constant variance in January and zero otherwise. Hence, we are effectively assuming a seasonal factor structure in which the number of factors in January (two) is different from the rest of the year (one), something which is in line with the empirical findings of Cho and Taylor (1987), and Connor and Korajczyk (1988). However, to ensure that the first factor is the same all year round, we do not divide our sample observations into January and rest of the year, but rather estimate our model using all months simultaneously. Finally, we assume that the factor loadings and prices of risk are time invariant. ${ }^{6}$

Hence, we consider the following model, in vector notation for the $N$ assets at hand and assuming that the common factors and idiosyncratic noises have a joint conditional normal distribution:

$$
\begin{gathered}
r_{t}=B \Lambda_{t} \tau+B f_{t}+\in_{t} \\
\left(\begin{array}{l}
f_{t} \\
\varepsilon_{t}
\end{array}\right) / I_{t-1} \sim N\left[\left(\begin{array}{l}
0 \\
0
\end{array}\right),\left(\begin{array}{cc}
\Lambda_{t} & 0 \\
0 & \Omega
\end{array}\right)\right]
\end{gathered}
$$

where $\Lambda_{t}=\operatorname{diag}\left\{\lambda_{1}, d_{t}\right\}, d_{t}=1$ for January and 0 otherwise, $f_{t}=\left(f_{1 t}, f_{2 t}\right) /$,

6. Notice, however, that for an alternative scaling of the factors with unit conditional variance, the model can also be interpreted as a time-varying factor betas model with constant variance factors (see Demos and Parissi [1998]). 
$f_{2 t}$ is 0 in non-January, $\tau=\left(\tau t_{1}, \tau_{2}\right) /$, and $\Omega=\operatorname{diag}\left\{\omega_{1}, \ldots, \omega_{N}\right\}$.

\section{The Large-N LM Test}

To clarify the Large-N LM ( $L L M)$ test, let us consider equation (3) but augmented by $m_{1}$ variables, say $x_{i t}$, which are specific to asset $i$ and $m_{2}$, say $y_{i}$, common to all assets. Let us call $C$ the $N \times\left(m_{1 N}+m_{2}\right)$ matrix of parameters, and $z_{t}=\left(x_{1}^{\prime}, \ldots, x_{N t}^{\prime}, y_{t}^{\prime}\right)^{\prime}$ the $\left(m_{1 N}+m_{2}\right) \times 1$ vector of predetermined variables. We would like to derive the LM test for the hypothesis that $C=0$. However, there are no closed form expressions for the first derivatives of the log-likelihood function and the Information Matrix is not block diagonal between the mean parameters and the factor conditional variance ones (see Demos and Vasillelis [1999]). Consequently, the $L M$ test would involve the regression of the residuals $\epsilon_{t}$ on the cross products of the variables in $z_{t}$, the estimated factors and their conditional variances, an excessively large number of variables.

However, if the common factors were observed the $k \times 1$ vector of factor representing portfolios $r_{f t}$, would be $\Lambda_{t} \tau+f_{t}$, so that $r_{t}=C z_{t}+B r_{f t}$ $+v_{t}$. Furthermore, if the conditional variances of the common factors do not depend on $B, C, \Omega$ and the parameters $(\tau, \psi)$ and $(B, C, \Omega)$ are variation free, we would have performed a sequential cut on the joint $\log$-likelihood function of $r_{t}, r_{f}$, which would make $r_{f t}$ weakly exogenous for $B, C$ and $\Omega$ (see Demos and Sentana [1998]). As a result, the unrestricted $M L$ estimates would be multivariate regression estimates, which, under the diagonality of $\Omega$, are simply obtained from $N$ univariate $O L S$ regressions of each $r_{i t}$ on $z_{i t}$ and $r_{f t}$. Then the LM is the usual one for $C_{i}=0$, i.e. it is $T R^{2}$ from the regression of the residuals $\epsilon_{i t}$ on $z_{i t}$ and $r_{f t}$ and it is distributed as $\chi^{2}$ with degrees of freedom the dimension of $z_{i t}$. Taking into account that the covariance matrix of residuals, $\Omega$, is assumed to be diagonal, the $L M$ test can be performed equation by equation (see Demos and Vasillelis [1999], for details).

To robustify, to departures from the normality assumption, the above $L M$ test, one can follow the 3-steps procedure of Wooldridge (1990). In the first step, the residuals, the vector of scores under the null (the factor representing portfolios in our case) and the misspecification indicators ( $z_{t}$ for our case) are standardized by some weights. ${ }^{7}$ The second step

7. In our case the weights are trivially one (see Davidson and MacKinnon [1985]). 
involves the regression of the elements in $z_{t}$ on the factor representing portfolios and save the residuals, say $z_{t}^{*}$. In the third step we regress 1 on the elements of $z_{t}^{*}$ multiplied by the $A P T$ residuals. The robust $L M$ test is $T R_{u}^{2}=T-S S R$, where $S S R$ is the Sum of Squared Residuals from the last regression. The test is asymptotically distributed as $\chi^{2}$ with degrees of freedom the dimensionality of $z_{t}$.

Unfortunately, the limiting factor representing portfolios are not observed and have to be estimated, in our case by the Kalman filter. However, as the number of assets increases, the estimated limiting portfolios converge to the true ones (see Demos and Sentana [1998], Grinblatt and Titman [1987], and Sentana [2002]) and consequently, the true limiting factors are revealed and are weakly exogenous. Hence, the $L M$ test of the previous paragraph applies. In that sense, the asymptotic distribution of the $L L M$ test is a $\chi^{2}$, requiring not only the time sample size to approach infinity, but the cross-sectional size, to increase as well. ${ }^{8}$ However, a complete analysis of $N$ and $T$ asymptotics is lacking. To assess the effect of parameter uncertainty, i.e. stemming from $T$ being finite, and large $N$ approximation on the $L L M$ test we perform a Monte Carlo exercise.

\section{A. Monte Carlo Simulations}

We generate 8000 samples of 250 observations each (plus another 250 for initialization) of a multivariate, with $N=7$, exact single factor model. Each of the seven returns is generated by:

$$
\begin{gathered}
r_{i, t}=\tau \beta_{i} \lambda_{t}+\beta_{i} f_{t}+v_{i, t} \\
\text { for } i=1, \ldots, 7
\end{gathered}
$$

where $f_{t} \sim N\left(0, \lambda_{t}\right), \lambda_{t}=0.25-0.23 f_{t-1}+0.1 f_{t-1}^{2}+0.65 \lambda_{t-1}, v i, t \sim N\left(0, \gamma^{2}\right)$ and $\gamma=\{1,5\}$, corresponding to high and low signal to noise ratios. ${ }^{9}$ The choices of the price of risk and factor loadings are the following: $\tau=$ $0.9, \beta_{1}=0.5, \beta_{2}=0.4, \beta_{3}=0.35, \beta_{4}=0.6, \beta_{5}=0.2, \beta_{6}=0.15$, and $\beta_{7}=$

8. In fact, it is possible to prove that, under appropriate conditions, even the $O L S$ factor scores are $\sqrt{N}$-consistent (see Sentana 2002).

9. As the unconditional variance of the factor is 1 , the variance of the idiosyncratic noise is what is known in the literature signal to noise ratio see, e.g., Harvey et al. (1992). 
0.21 . Notice that, all the aforementioned parameter values and with low signal to noise ratio, i.e., $\gamma=5$, roughly match the results in the empirical application section. We further simulate the variables $x_{1 t}=$ $0.9 x_{1 t-1}+0.7 f_{t}+\epsilon_{1 t}, x_{2 t}=-0.5 x_{2 t-1}+0.3 f_{t}+\epsilon_{2 t}$ and $x_{3 t}=0.8 x_{3 t-1}+\epsilon_{3 t}$ where $\left(\epsilon_{1 t}, \epsilon_{1 t}, \epsilon_{1 t}\right) / \sim N\left(0, I_{3}\right)$, representing two "economic" variables in the spirit of King et al. (1994) and one additional which is completely unrelated to returns.

As the estimation of latent factor heteroskedastic models is very time consuming (see also Demos and Sentana [1998], Lin [1992], and Sentana and Fiorentini [2001]), to estimate the factor via the Kalman filter for the above multivariate model, even with only seven assets, in each of the 8000 replications is a formidable task. However, the objective of these simulations is to incorporate two sources of uncertainty. One is associated with the estimation of the factor and consequently, with the factor representing portfolio, i.e., the finite $N$ source of uncertainty. The second is associated with the estimation of the parameters of the model, i.e., the finite $T$ source of uncertainty. In fact, in each replication we estimate the limiting factor portfolio by GLS, i.e. $r_{f_{t}^{G}}=\left(\widehat{\beta}^{\prime} \hat{\Gamma}^{-1} \hat{\beta}\right)^{-1} \hat{\beta}^{\prime} \hat{\Gamma}^{-1} r_{t}$ where the hat denotes the $O L S$ estimates from the regressions of the individual asset returns on the simulated factor representing portfolio, thus incorporating both sources of uncertainty. Notice that according to Sentana (2002), the GLS limiting portfolio estimator is, for any fixed $N$, less efficient as compared to the Kalman filter one. In fact, the relative unconditional efficiency is, in our case, 0.04 and 0.5 , respectively for the low signal to noise ratio for the high one. Hence, employing the conditional Kalman filter to estimate the factor has considerable efficiency gains. This is very important as one expects that the size distortion of the LLM test employing the conditional Kalman filter scores will be much lower as compared with the one employing $f_{t}^{G}$.

Under the null hypothesis a constant, $x_{1 t}, x_{2 t}$ and $x_{3 t}$ should have zero predictive power on the residuals from the regressions of returns on the estimated $G L S$ portfolio, $r_{f_{t}^{G}}$. Consequently, the robust $L L M$ test for each individual asset should be distributed as $\chi_{4}^{2}$, whereas under the hypothesis of diagonal idiosyncratic variance, their sum should be distributed as $\chi_{28}^{2}$. The $p$-value discrepancy plots proposed by Davidson and MacKinnon (1998) are employed to display the simulation evidence on the size of the tests. Figure 1 presents the size distortions of the LLM 


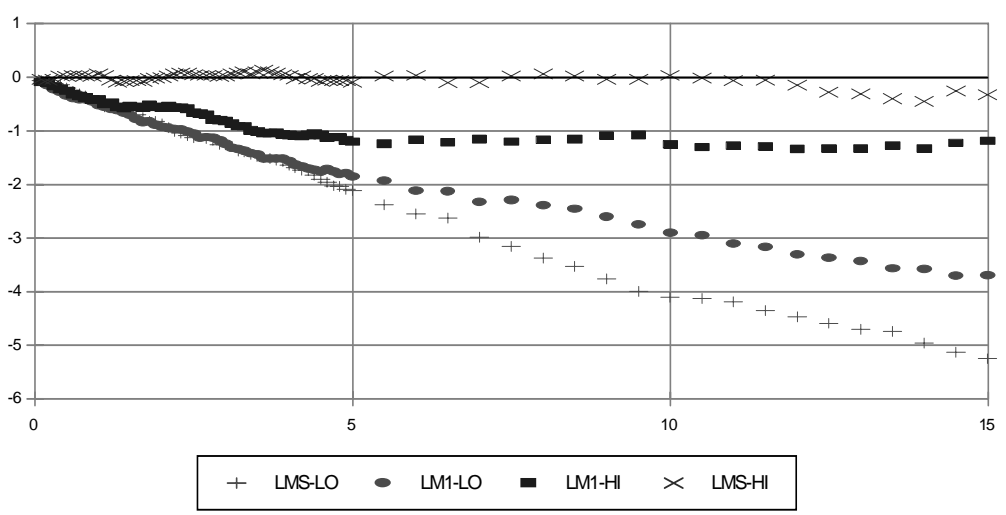

FIgURE 1.- $P$-value Discrepancy Plots

test for the first individual asset, $L M 1-H I$ and $L M 1-L O$, and the sum of the $L L M$ tests for all individual assets, $L M S-H I$ and $L M S-L O$, for high and low signal to noise ratio. The largest size distortion is observed for the sum of individual $L L M$ tests when the signal to noise ratio is low, whereas the sum of individual $L L M$ tests when the signal to noise ratio is high is very close to the nominal size.

To investigate the power of the $L L M$ test, we simulate the seven returns by the following process:

$$
\begin{gathered}
r_{i, t}=0.1+x_{1 \mathrm{t}-1}+x_{2 t-1}+x_{3 t-1}+\tau \beta_{i} \lambda_{t}+\beta_{i} f_{t}+v_{i, t} \\
\text { for } I=1, \ldots, 5
\end{gathered}
$$

where the processes $x_{1}, x_{2 t}, x_{3 t}$, and $\lambda_{t}$ are generated as before, and the parameter values of $\tau$ and the $\beta_{j}^{\prime} s$ are as above. The evidence for the power of the four tests is presented in figure 2 using the Davidson and MacKinnon (1998) size-power curves. The least powerful test is, as expected, the individual $L L M$ test when the signal to noise ratio is low. However, notice that although in this section the factor scores are estimated by $G L S$ in the application they are estimated by the Kalman filter with time varying weights. As now the relative efficiency of the two estimators is at least 0.04 it is expected that the individual Kalman filter $L L M$ tests will be considerable less size distorted and more powerful than the $G L S$ ones. 


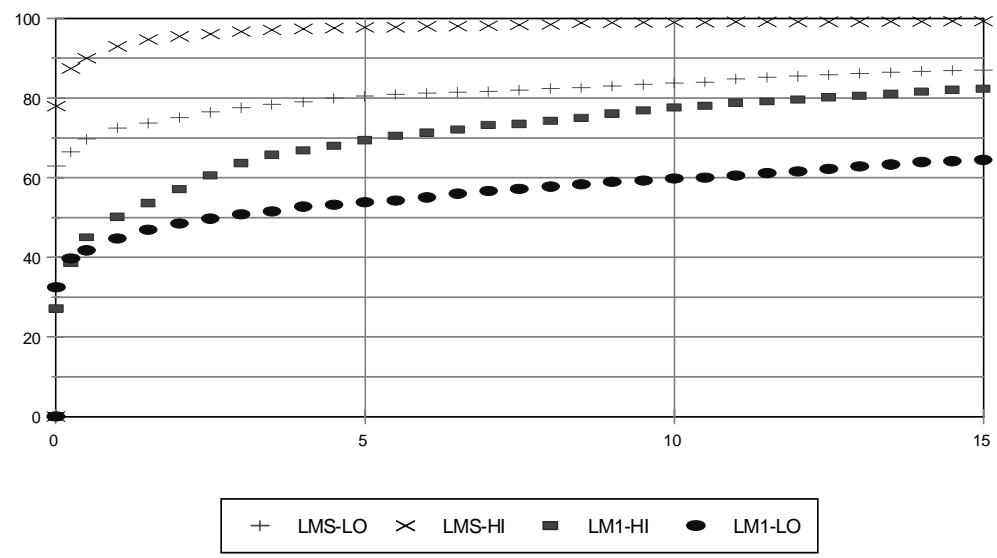

FIGURE 2.- Size-Power Curves

\section{Empirical Application}

We consider all individual excess returns from stocks continuously quoted in FTSE 100 Index of the London Stock Exchange throughout our sample period 1973:3 to 1992:6. This results in a number of 45 individual companies, and each of them contains 232 observations across time. ${ }^{10}{ }^{11}$ All data are collected from Datastream. We consider as a safe rate the 1-month T-bill rate. ${ }^{12}$ Let us first consider the predictability of the accounting and economic variables.

\section{A. Predictability of Instruments}

We extract 16 canonical factors, which explain $86.6 \%$ of the maximum correlation between these instruments and the 45 excess returns under consideration. Our approach is more time efficient than the MPP of Lo and MacKinlay (1997) as it skips the step where individual returns are

10. This selection could introduce survivorship biases. However, any other selection criterion would result in lack of data on the accounting variables.

11. See Demos and Vasillelis (1999) for the names of individual companies.

12. Updating the dataset is not a simple task. Apart from the fact that there is no access to Datastream any more, the main difficulty is that some of the companies are not quoted anymore at the London Stock Exchange as they have, probably, merged. 
regressed on the instruments. In any other respect both techniques are identical (see Demos and Vasillelis [1999]). The first factor explains more than $57 \%$ of the $M P P$, whereas the $26^{\text {th }}$ factor explains only $1.6 \%$ of the least predictable portfolio. To specify the number of significant canonical correlations, in our case, is not a simple task. This is because first the number of instruments is quite large, and consequently any attempt to test for the number of significant factors is bound to fail due to the excessively large number of degrees of freedom. ${ }^{13}$ Second, in our sample there are at least three variables which are considered nonstationary. Even after taking into account our maintained hypothesis, i.e., that there exists at least one cointegrating relationship, this would possibly result in further deterioration of the power of any statistical test. Bearing also in mind the results in Foster et al. (1997), Lo and MacKinlay (1997) and Ang and Bekaert (2001) we decided to specify the number of canonical factors to 16, thus hoping to avoid first any statistical artifacts which may result from data mining, second any spurious results from the nonstationary variables, and third possibly reduce the small-sample biases. ${ }^{14}$

Let us now turn our attention to the prediction results of equation (1) in section 2 . As we are looking for the highest $R^{2}$ we use equation by equation $O L S$ procedure. $R^{2}$ 's range from $17.71 \%$ to $36.00 \%$ with an average value, over the 45 regressions, of $28.18 \%$, whereas the equivalent $\bar{R}^{2}$ 's (adjusted for the number of explanatory variables) range from $7.72 \%$ to $28.22 \%$ with an average value of $19.46 \%$ (for complete results see Demos and Vasillelis [1999]). For monthly data, these $R^{2}$ s are much larger than the ones reported in the literature (see e.g. Campbell [1987], Fama [1991], Ferson and Harvey [1991], Keim and Stambaugh [1986], Lo and MacKinlay [1997], Sentana and Wadhwani [1991] etc.), and they become more impressive when one considers that we examine excess returns of individual companies, which in general are noisier than portfolio ones. However, this is the first time, at least to our knowledge, that such an extensive dataset of

13. For example, the test that the last 11 canonical correlations are jointly zero has a $\chi^{2}$ distribution with 319 degrees of freedom (see Anderson [1984]).

14. One weakness any statistically motivated approach such as canonical correlation, principal components or factor analysis is that the factor weights are not scale free. Hence, any attempt in investigating the importance of each one of the economic variables would be ambiguous. Furthermore, the results in Stambaugh (1999) and Ang and Bekaert (2001) cast additional doubt on the interpretation of the factors. 
instruments, including both economic and accounting variables, is employed.

One should be careful how to interpret these results under the light of Stambaugh (1999), although for monthly data the biases should be of small order (see Ang and Bekaert [2001]). Furthermore, whether these results represent genuine predictability or are statistical artifacts (see Lo and MacKinlay [1990]), is a matter of consideration. From the results in Lo and MacKinlay (1997), it seems that what is important for the critical values of maximum $R^{2}$ is the number of observations (see also Lo and MacKinlay [1995]). However, notice that these critical values are for 6 predictive variables, a number which falls short of 26 , the number of instruments in our case. Up to what degree this difference will affect the $R^{2}$ critical values is not known. Nevertheless, it is only natural that our choice of variables, either economic or accounting, is driven by theoretical as well as empirical arguments. In fact this is the main scope of this paper, i.e., to identify if there is any "true" predictability coming from a wide range of accounting and economic variables. This, however, introduces pre-test biases in the terminology of Lo and MacKinlay (1990). Consequently, it is difficult, if not impossible, to avoid this fact, especially on a topic such as the $E M H$.

\section{B. The Seasonal Latent Factor Model}

As we consider 45 assets, which is, to our knowledge, the largest number of assets where a model with time varying conditional first and second moments is estimated, the total number of parameters is $140{ }^{15}$ Hence, to speed up the estimation procedure, we employ the $E M$ (Expectation-Maximization) algorithm suggested in Demos and Sentana (1998) to obtain good starting values for a standard quadratically convergent method. Estimating the conditional $A P T$ model in equations 3 and 4, we get factor loadings that they range from 4.67 to 9.17 and -7.20 to 9.65 for the first and second factor, respectively. The cross-sectional variance of the betas for the first factor is 0.96 , something which is very important for the mean square convergence of the limiting factor representing portfolio. The values of two prices of risk are 0.05 , for the whole year one, and 0.12 for the January one, both

15. Whereas traditional applications of factor analysis on asset returns often use a large collection of assets, those that take into account variation in conditional moments have considered a much smaller number of assets (see Ng et al. [1992], King et al.[1994], Sentana [1995] etc.). This is because the estimation of such models is very time-consuming. 


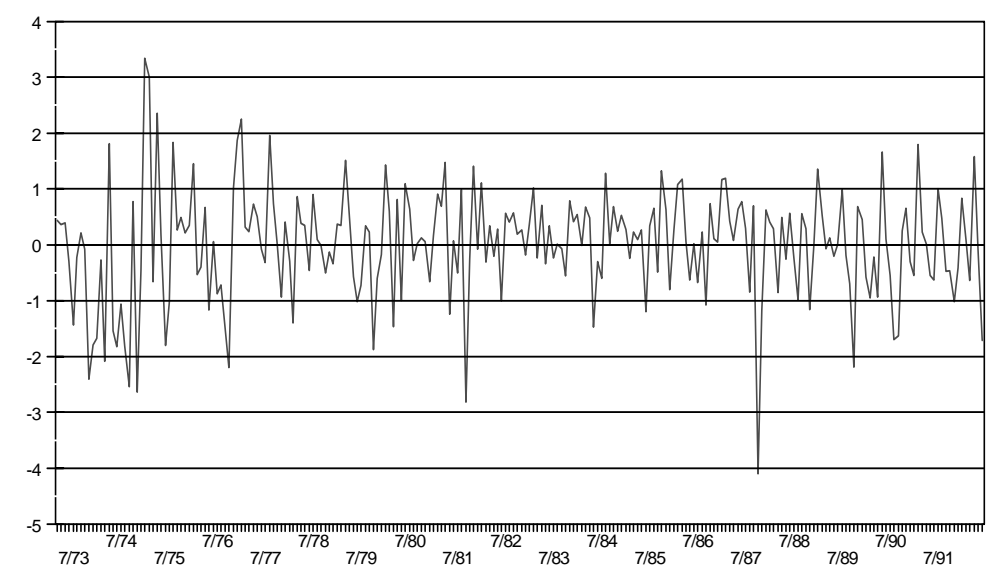

FIGURE 3.- Whole-Year-Factor Scores

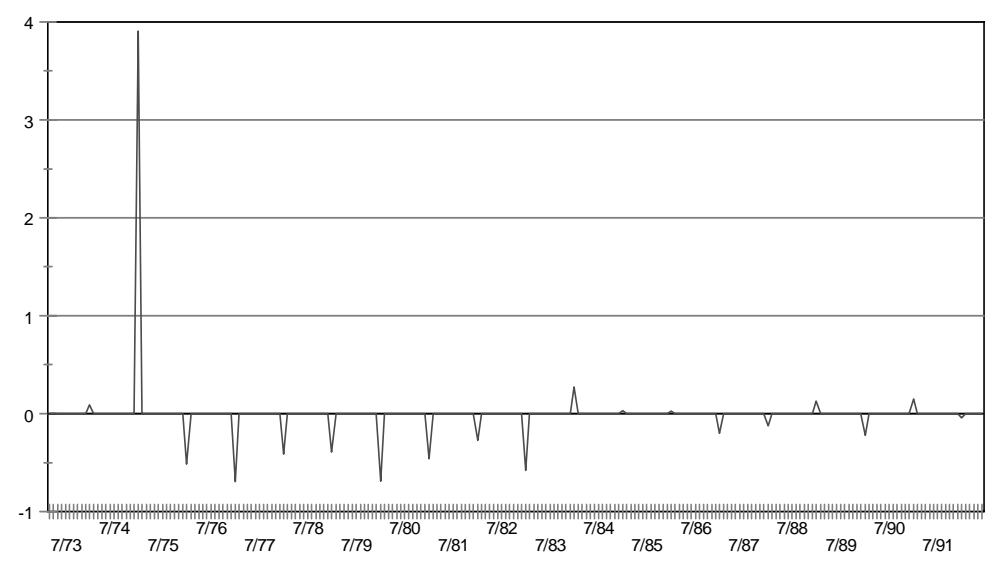

FIGURE 4.- January-Factor Scores

positive, with $t$-statistics of 0.74 and 0.45 , respectively (analytical results are available upon request). These values are considered very small, which indicates that these variables are imprecisely estimated, something which is not new in applied work (see King et al. [1994]). This, however, is mainly due to the noise in the data. Furthermore, what is important for our question is the time variation of the risk premium, i.e. $\beta_{i 1} \lambda_{1 t} \tau_{1}+\beta_{i 2} d_{t} \tau_{2}$, and not the values of the prices of risk. 


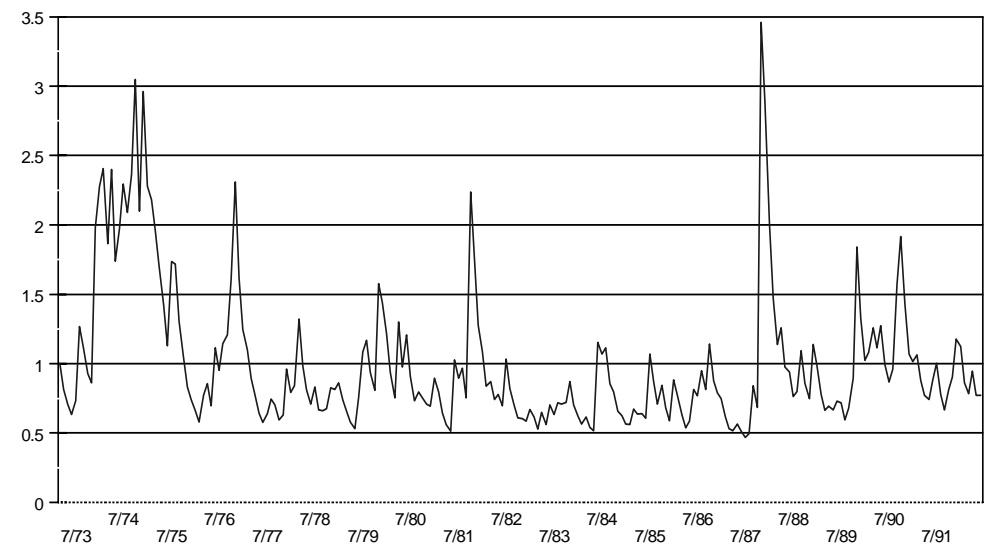

FIGURE 5.-Whole-Year-Factor Conditional Variance

In figures 3 and 4 we present the estimated all-year and January factors, respectively. For the first figure the crash of October 87 stands out, whereas the January factor exhibits a positive return of $3.9 \%$ in January 1975. Figure 5 presents the estimated conditional variance of the first factor. In accordance with the results in Sentana (1995) for the FT500, there seems to be a fair amount of variation over time and a fair degree of persistence (estimated value 0.752 ). Two periods of increased volatility are apparent: the pre-January 1975 period and the October $1987 \mathrm{crash}$. There is also evidence of dynamic asymmetric, leverage, effect (estimated value $-0.226, L R=10.22, p$-value $=0.001$, see table $1)$.

A number of misspecification Likelihood Ratio tests are presented in table 1. The test for including constants in equation 3 is 27.24. Consequently, the null hypothesis of zero intercepts cannot be rejected at $5 \%$ level $(p$-value $=0.98)$. This is an important result, as in the opposite case it could be claimed as evidence of anyone or all of the following facts. First, the estimated limiting factor representing portfolio is estimated inefficiently (see Gibbons et al. [1989]). However, using the Kalman filter to estimate the factor, this possibility is rather low. Second, as we assume constant idiosyncratic variances, the existence of significant constants could be an indication of idiosyncratic risk pricing. In any case, rejecting the null of zero intercepts would indicate model failure, as it would imply that it does not price assets 
TABLE 1. APT Tests

\begin{tabular}{lcc}
\hline \multicolumn{1}{c}{ Test } & Value & Critical Value under $\mathrm{H}_{0}$ \\
\hline$\psi_{1}=0$ & 10.22 & $\chi_{1,5 \%}^{2}=3.84$ \\
Constants in the mean & 27.24 & $\chi_{45,5 \%}^{2}=61.65$ \\
Proportionality & 131.80 & $\chi_{45,5 \%}^{2}=61.65$ \\
Unconstrained $\tau_{1}$ & 40.38 & $\chi_{44,5 \%}^{2}=60.60$ \\
Unconstrained $\tau_{2}$ & 74.68 & $\chi_{44,5 \%}^{2}=60.60$ \\
Ljung-Box $Q$-test of & & \\
$f_{1 t} / \sqrt{\lambda_{1 t}}$ & 6.88 & $\chi_{12,5 \%}^{2}=21.0$ \\
Ljung-Box Q-test of & & \\
{$\left[f_{1 t} / \sqrt{\lambda_{1 t}}\right]^{2}$} & 3.27 & $\chi_{12,5 \%}^{2}=21.0$ \\
Normality of & & \\
$f_{1 t} / \sqrt{\lambda_{1 t}}$ & 72.16 & $\chi_{2,5 \%}^{2}=5.99$
\end{tabular}

Note: Tests on the following APT model:

$$
r_{t}=\left(\beta_{1}, \beta_{2}\right)\left[\begin{array}{cc}
\lambda_{1 t} & 0 \\
0 & d_{t}
\end{array}\right]\left(\begin{array}{l}
\tau_{1} \\
\tau_{2}
\end{array}\right)+\left(\beta_{1}, \beta_{2}\right)\left(\begin{array}{l}
f_{1 t} \\
f_{1 t}
\end{array}\right)+v_{t}
$$

where $d_{t}$ is 1 for January and 0 otherwise and

$$
\lambda_{1 t}=\psi_{0}+\psi_{1} E_{t-1}\left(f_{1 t-1}\right)+\psi_{2} E_{t-1}\left(f_{1 t-1}^{2}\right)+\psi_{3} \lambda_{1 t-1} .
$$

correctly on average. ${ }^{16}$ Third, the existence of a missing priced factor. ${ }^{17}$

The January effect can be attributed to the seasonality in the conditional variance of a single factor (see Ferson and Harvey [1991]). In our setup, this would mean 45 testable restrictions, i.e., that for each asset, the factor loading of the first factor is proportional to the factor loading of the second factor with the same proportionality factor as that of the two prices of risk $\left(\tau_{2}=\theta_{\tau 1}\right.$ and $\beta_{i 2}=\theta \beta_{i 1}$ for $\left.i=1, \ldots, N\right)$. However, the Likelihood Ratio strongly rejects this hypothesis (a value of 131.80, $p$-value $\left.=2 * 10^{-10}\right)$. Consequently, we can say that the U.K. stock

16. However, notice that this is not the case when the factors are approximated by other methods (see, e.g., Fama and French [1996]).

17. However, even in this case, under some conditions, an APT model still applies (see Middleton and Satchell [1999]). 
exchange is characterized by the fact that not only is the risk premium higher in January in absolute terms, but that the cross-sectional structure of returns is affected as well. This is in contrast to the U.S. experience (see Ferson and Harvey [1991]).

The conditional $A P T$ model in equations 3 and 4 has two cross equation restrictions, i.e., the prices of risk, $\tau_{1}$ and $\tau_{2}$, are common across assets. The likelihood ratio for unrestricting $\tau_{1}$ is 40.38 , which is insignificant at $5 \%$ level ( $p$-value $=0.63$ ). However, unrestricting $\tau_{2}$ produces a likelihood ratio statistic of 74.68 , which is significant at the same level ( $p$-value $=0.002)$. Consequently, we can conclude that the price of risk is common to all assets all year round, apart from January. This rejection of the common price of risk for January is another feature that differentiates the U.K. from the U.S. stock market, as in the latter the risk-return relationship is stable across assets only for the January returns. One possible explanation is that the factor for January, as it is modeled here, is not a fully seasonal factor, in the sense that for the other months of the year its value is 0 . Although the hypothesis of common January price of risk is rejected by the data, we keep this restriction, as in this way we can claim that what we extract from the individual asset excess returns is a January risk premium, something which is in line with the $A P T .^{18}$

Finally, we test the assumptions postulated by equation (4). Employing the Ljung-Box $Q$ test the hypotheses that the standardized latent factor and its square are uncorrelated up to $12^{\text {th }}$ order are not rejected at $5 \%$ level $(p$-value $=0.865)$ and $(p$-value $=0.993)$, respectively. However, the normality of this random variable, as measured by the Jarque-Bera test, is rejected ( $p$-value $=0.000$ ). Furthermore, the $A P T$ residuals are not normally distributed, at 5\% level, in 34 out of the 45 cases. Nevertheless, the non-normality of the standardized factor and the $A P T$ residuals do not affect the distribution of the robust $L L M$ test.

\section{The LLM Test Results}

Let us now consider the residuals $\hat{\varepsilon}_{t}$ from our seasonal latent factor

18. The closest to our model with the U.S. data is the factor GARCH employed by $\mathrm{Ng}$ et al. (1992), where they consider 3 factors one of which is closely related to a January dummy. However, they do not test for the extra cross equation restriction implied by this factor. 
TABLE 2. Robust $L L M$ Test

\begin{tabular}{llll}
\hline Stc. No & $L L M$ & Stc. No. & LLM \\
\hline 1 & 32.95 & 24 & 32.62 \\
2 & 34.95 & 25 & 29.56 \\
3 & $41.15^{*}$ & 26 & $40.01^{*}$ \\
4 & $40.71^{*}$ & 27 & 25.69 \\
5 & $39.82^{*}$ & 28 & $43.57^{*}$ \\
6 & 30.19 & 29 & $39.56^{*}$ \\
7 & 31.90 & 30 & 22.06 \\
8 & 31.61 & 31 & 32.68 \\
9 & $40.26^{*}$ & 32 & 31.58 \\
10 & 33.73 & 33 & $39.82^{*}$ \\
11 & 34.46 & 34 & 25.25 \\
12 & $44.33^{*}$ & 35 & $43.37^{*}$ \\
13 & 21.97 & 36 & $44.19^{*}$ \\
14 & 28.13 & 37 & 33.23 \\
15 & 28.11 & 38 & 34.57 \\
16 & 34.70 & 39 & 35.22 \\
17 & $40.18^{*}$ & 40 & 22.09 \\
18 & 37.73 & 41 & 24.04 \\
19 & 28.17 & 42 & 32.24 \\
20 & 29.67 & 43 & 27.90 \\
21 & $39.42^{*}$ & 44 & 26.52 \\
22 & 29.21 & 45 & 36.12 \\
23 & 34.95 & & \\
\hline & & &
\end{tabular}

Note: Robust $L L M$ test for 45 individual shares $\chi_{26,5 \%}^{2}=38.8, \chi_{26,1 \%}^{2}=45.6$

(*) significant at $5 \%$.

model (see equations (3) and (4)). It is obvious that any predictability of the two risk premia has been extracted. Consequently, if there is any predictability from the economic and/or accounting variables it could be attributed to either anyone or all of the following reasons. First, the adopted asset pricing model does not hold, i.e., it does not approximate risk premia adequately. Second, there are indeed inefficiencies in the market. On the other hand, if these potentially predictive variables do not have any power on the residuals, their initial predictability on excess returns was due to the fact that these variables approximate risk premia. To resolve the above question we employ the robust $L L M$ test of section IV.

In table 2 we present the robust $L L M$ test for the 45 individual asset excess return. In 13 out of 45 cases we reject, at $5 \%$ size, the null hypothesis of zero coefficients for the predictive variables, whereas this 
number reduces to 0 for a $1 \%$ size test. It could be claimed that these results are due to the fact that the robust $L L M$ rejects the null when the $R^{2}$ is high to start with and vice versa. However, the correlation between the two is only 0.29 . Consequently, it seems that $R^{2}$ and robust LLM values are correlated only mildly.

In the light of these results we can say that there is no predictability of the accounting and economic instruments in $71.1 \%$ of the individual stocks. This of course is not very impressive especially if one considers that the expected number is $95 \%$. However, there are two points that could explain these results. First, the distribution of the robust $L L M$ test could be affected by the biases of the coefficients (see Stambaugh [1999]). Second, notice that the procedure applied to form the canonical variates uses the whole sample of 232 observations, something which is of great importance as existing evidence supports the time variation in the factor weights (see also Lo and MacKinlay [1997]). In such a case, the whole testing procedure is in favor of the predictive variables, as the latent factor model is implicitly a time varying parameter model (see section III). Of course the same can be claimed for the accounting variables, the January dummy and the constant. However, the Chow tests, breaking the sample of 232 observations into 2 subsamples of 132 and 100 observations respectively, reject the null in only 8 cases out of 45 at $5 \%$ and 3 at $1 \%$.

To investigate this issue we evaluated the 16 canonical factors for the 150 last observations recursively, i.e. starting from the $82^{\text {nd }}$ observation we form the factor scores for time $t$ by using the estimated weights from periods 1 to $t-1$. Repeating the $O L S$ regressions of the excess returns on the accounting and the 16 recursively estimated factors, the average $R^{2}$ is now $25.32 \%$ and the average $\bar{R}^{2}$ is $10.27 \%$, both lower than previously when the weights for the canonical factors were estimated from the full sample. ${ }^{19}$

In terms of the robust $L L M$, at size $5 \%$, we now have only 2 cases where the null is rejected, whereas at $1 \%$ we do not reject in any of the 45 individual cases (see table 3). Consequently, we can say that in at least $95.5 \%$ of the cases in the U.K. stock market, the latent factor $A P T$ model considered here explains excess returns better than a constant, a January dummy, the 8 accounting and 16 economic canonical factors.

19. Employing recursive estimation and predict the weights to form the factor scores will also reduce the bias problem. 
TABLE 3. Robust $L L M$ Test Forecasting the Canonical factor

\begin{tabular}{llcl}
\hline Stc. No. & $L L M$ & Stc. No. & $L L M$ \\
\hline 1 & 29.42 & 24 & 23.81 \\
2 & 29.25 & 25 & 31.18 \\
3 & 22.43 & 26 & 29.04 \\
4 & 36.82 & 27 & 34.17 \\
5 & 27.28 & 28 & 23.25 \\
6 & 31.02 & 29 & 33.08 \\
7 & 22.77 & 30 & 25.36 \\
8 & 26.60 & 31 & 30.56 \\
9 & 29.31 & 32 & 28.71 \\
10 & 26.28 & 33 & 33.40 \\
11 & 31.09 & 34 & 22.81 \\
12 & 28.11 & 35 & 30.36 \\
13 & 28.75 & 36 & 28.03 \\
14 & 28.55 & 37 & 32.91 \\
15 & 33.57 & 38 & 33.43 \\
16 & 33.01 & 39 & 34.48 \\
17 & 25.12 & 40 & 18.32 \\
18 & 30.36 & 41 & 25.39 \\
19 & 27.78 & 42 & 32.21 \\
20 & 34.83 & 43 & 19.08 \\
21 & $40.07 *$ & 44 & 25.03 \\
22 & 24.69 & 45 & 36.28 \\
23 & $39.27 *$ & & \\
& & &
\end{tabular}

Note: Robust $L L M$ test for 45 individual shares. Forecasting the canonical factor $\chi_{26,5 \%}^{2}=38.8, \chi_{26,1 \%}^{2}=45.6,(*)$ significant at $5 \%$.

Hence, taking into account also that the latent factor model is a relatively simple one, with only one all year factor and an additional for January, these results indicate that the predictability observed in the U.K. stock market is probably due to risk premia and not due to inefficiencies. Alternatively, it could be the case that the power of the individual robust $L L M$ tests are very low, although the simulations in section IV B indicate that this is not the case.

To investigate this issue a bit further, we test the null hypothesis that the $A P T$ residual predictability, of the 26 instruments considered here, is zero jointly for the 45 individual cases. Notice that the joint test is in principal more powerful, something which is also confirmed by the simulation exercise (section IV A). Under the maintained assumption of an exact 2 latent factor seasonal model the residual idiosyncratic covariance matrix is diagonal. Consequently, the robust $L L M$ tests are 
independent. Applying the Central Limit Theorem for identically and independently distributed random variables we get that the sample mean, standardized appropriately, has, under the null, a standard normal distribution. However, in our case the statistic takes the value of 3.04 ( $p$-value $\left.=1.2 * 10^{-3}\right)$, which highly rejects the joint hypothesis. This casts some doubt on the power of the individual asset robust $L L M$ tests.

Alternatively, this result could be attributed to the fact that the number of cases, 45 , is not big enough to allow the standardized sum of independent $\chi_{26}^{2}$ random variables to approximate the standard normal distribution adequately. To investigate this issue we generated 8000 samples of 45 independent random variables each distributed as $\chi_{26}^{2}$ and derived the distribution of their standardized sum. The resulting $p$-value for 3.04 is $1.4^{*} 10^{-2}$, which again rejects the null hypothesis at $5 \%$ but not at $1 \%$.

Furthermore, it could be the case that the rejection of the joint test is due to the fact that we impose the restriction of common price of risk for the January factor, $\tau_{2}$, a restriction which has been rejected by the data (see previous subsection). Unrestricting $\tau_{2}$ and forming the joint $L L M$ statistic we get a value of 2.73 (simulated $p$-value $=2.3 * 10^{-2}$ ), again rejecting the null at $5 \%$ but not at $1 \%$. Consequently, we can say that the restriction of common price of risk for the seasonal factor only marginally affects the rejection of the joint test. Finally, it could be the case that $\Omega$ is not diagonal or that the "true" number of factors is more than two. In the first case the joint $L L M$ test is not the sum of individual ones whereas in the second, provided that the missing factors and predictive variables are not correlated, the $L L M$ test should not be affected. We leave these issues for the future.

\section{Further Research and Conclusions}

This paper contributes to the $E M H$ issue, by suggesting a three-step procedure to investigate whether the observed predictability in stock returns is due to inefficiencies or due to the risk premium structure. The procedure is applied to monthly individual stock excess returns from companies quoted at the London Stock Exchange. First, we document impressive levels of excess return predictability by a large variety of lagged economic and asset specific accounting variables. Second we employ a seasonal latent factor $A P T$ model which on average prices assets correctly. Two important differences are revealed between the 
U.S. and U.K. stock markets: in the case of the U.S., the risk premium is higher in January without affecting the factor structure, and the price of risk for January factor is common across all assets. None of these is true for the U.K.

On the third step we employ the robust, to departures from our distributional assumptions, $L M$ principle, to test whether the economic and accounting variables, considered in the first step, have any predictive power on the residuals of the asset pricing model. The application of the $L M$ test reveals that in $95.5 \%$ of the cases considered here the economic and accounting instruments have no additional significant predictability. However, testing the null for all cases jointly results in rejecting the hypothesis at $5 \%$ level but not at $1 \%$. Although this rejection can be attributed to a number of reasons, including the power of the individual tests and/or statistical artifacts, it demonstrates once more that the largest part of the original documented predictability, if not all, is attributed to the risk premium structure.

The presented results are important for both academics and practitioners as they could constitute empirical justification of the parable in Ferson et al. (1999), i.e., variables that seem important to the cross-section of stock returns will appear to be useful risk factors, but their importance might disappear if risk premia are measured using a better procedure. Furthermore, the estimation of any asset pricing model should involve the interdependence of time varying first and second moments of asset returns and the factor should be estimated efficiently, an issue which is not referred to by the theoretical models but by their empirical implementations.

However, this paper leaves open questions, on a theoretical as well as an empirical level. For example, the important differences between two well-developed stock markets, namely the U.K. and U.S., during January cannot be accommodated within any of the well-known asset pricing models. On the other hand, modeling excess asset returns by applying a latent factor $A P T$ type model is not without problems. The rejection of the common price of risk, across assets, for January is an indication of this. Furthermore, there are many "competing" processes to model conditional time variation of second moments in the literature. Which of these produces the best results has not yet been examined. 


\section{Appendix}

TABLE A.

\begin{tabular}{ll}
\hline & \multicolumn{2}{c}{ Economic Variables } \\
\hline \% Changes of Exchange Rate & Unexpected Inflation \\
FTALL Index Dividend Yield & Expected Inflation \\
$\Delta$ in FTALL Index Dividend Yield & Production \\
FTALL Excess Return & Production Growth $_{t-1}$ \\
3-Month T-Bill Rate & Production Growth $_{t-2}$ \\
20-Year Bond Yield & Unexpected Production Growth \\
Changes in 1-Month T-Bill & Expected Production Growth \\
Changes in 3-Month T-Bill & Annual Production Growth \\
Changes in 20-Year Yield & M0 \\
Term Spread & M0 Growth \\
Price Index & M0 Growth \\
Inflation $_{t-1}$ & Unexpected M0 Growth \\
Inflation $_{t-2}$ & Expected M0 Growth \\
\hline
\end{tabular}

TABLE B.

Accounting Variables

Own Dividend Yield

Log Market Value

Price/Earnings

Price/Sales

Price/Cash Flow

Market Value/Book Value

Borrowing Ratio

Working Capital Ratio

\section{References}

Al-Qenae, R.; Li, C.; and Wearing, B. 2002. The information content of earnings on stock prices: The Kuwait exchange. Multinational Finance Journal 6:197-221.

Anderson, T. W. 1984. An introduction to multivariate statistical analysis J. Wiley \& Sons.

Ang, A., and Bekaert, G. 2001. Stock return predictability: Is it there? Discussion Paper, Columbia University.

Campbell, J. Y. 1987. Stock returns and the term structure. Journal of Financial Economics 18:373-399. 
Campbell, J. Y., and Hentschel, L. 1992. No news is good news: An asymmetric model of changing volatility in stock returns. Journal of Financial Economics 31:283-318.

Campbell, J. Y., and Shiller, R. J. 1988. The dividend-price ratio and expectations of future dividends and discount factors. Review of Financial Studies 1:195-228.

Chamberlain, G., and Rothschild, M. 1983. Arbitrage, factor structure, and mean-variance analysis on large asset markets. Econometrica 51:1281-1304.

Chan, L. K. C.; Hamao, Y.; and Lakonishok J. 1991. Fundamentals and stock returns in Japan. The Journal of Finance 46: 1739-1764.

Cho, D. C., and Taylor, W. M. 1987. The seasonal stability of the factor structure of stock returns. The Journal of Finance 42:1195-1211.

Connor, J., and Korajczyk, R. 1988. Risk and return in an equilibrium APT: Application of a new test methodology. Journal of Financial Economics 21:255-289.

Davidson, R., and MacKinnon, J. G. 1985. Heteroskedasticity-robust tests in regression directions. Annales de l'INSEE 59:183-218.

Davidson, R., and MacKinnon, J. G. 1998. Graphical methods for investigating the size and power of tests statistics. The Manchester School 66:1-26.

Demos, A., and Parissi, S. 1998. Testing asset pricing models: The case of Athens stock exchange. Multinational Finance Journal 2:189-223.

Demos, A., and Sentana, E. 1998. An EM algorithm for conditionally heteroskedastic factor models. Journal of Business and Economic Statistics 16:357-361.

Demos, A.; Sentana, E.; and Shah, M. 1994. Risk and return in January: Some U.K. evidence. In J. Kaehler and P. Kugler (eds). Econometric Analysis of Financial Markets Physica Verlag, 185-201.

Demos, A., and Vasillelis, G. 1999. U.K. stock market inefficiencies and the risk premium, Discussion Paper 99-12. Dept. of International and European Economic Studies, Athens University of Economics and Business.

Diebold, F., and Nerlove, M. 1989. The dynamics of exchange rate volatility: A multivariate latent factor ARCH model. Journal of Applied Econometrics $4: 1-21$.

Engle, R. F. 1987. Multivariate ARCH with factor structures. Cointegration in variance. Discussion Paper. University of California at San Diego.

Fama, E. F. 1981. Stock returns, real activity, inflation, and money. The American Economic Review 71:545-565.

Fama, E. F. 1991. Efficient capital markets: II. The Journal of Finance 46:1575-1617.

Fama, E. F., and French, K. R. 1988. Dividend yields and expected stock returns. Journal of Financial Economics 22:3-25.

Fama, E. F., and French, K. R. 1996. Multifactor explanations of asset pricing anomalies. The Journal of Finance 51:55-84. 
Fama, E. F., and Schwert, W. 1977. Asset returns and inflation. Journal of Financial Economics 5:115-146.

Ferson, W. E., and Harvey, C. R. 1991. The variation of economic risk premiums. Journal of Political Economy 99:385-415.

Ferson, W. E.; Sarkissian, S.; and Simin, T. 1999. The alpha factor asset pricing model: A parable. Journal of Financial Markets 2:49-68.

Foster, D. F.; Smith, T.; and Whaley, R. E.1997. Assessing goodness-of-fit of asset pricing models: The distribution of the maximal $R^{2}$. The Journal of Finance 52:591-607.

Gibbons, M. R.; Ross, S. A.; and Shanken, J. 1989. Tests of the efficiency of a given portfolio. Econometrica 57:1121-1152.

Grinblatt, M., and Titman, S. 1987. The Relation between mean-variance efficiency and arbitrage pricing. Journal of Business 60:97-112.

Gultekin, M. N., and Gultekin, N. B. 1983. Stock market seasonality: International evidence. Journal of Financial Economics 12:469-481.

Harvey, A.; Ruiz, E.; and Sentana, S. 1992. Unobservable component time series models with ARCH $\backslash$ disturbances. Journal of Econometrics 52:129-158.

Harvey, C. R. 1991. The world price of covariance risk. The Journal of Finance 46:111-157.

Huberman, G.; Kandel, S.; and Stambaugh, R. F. 1987. Mimicking portfolios and exact arbitrage pricing. The Journal of Finance 42:1-9.

Jermakowicz, E., and Gornik-Tomaszewski, S. 1998. Information content of earnings in the emerging capital market: Evidence from the Warsaw stock exchange. Multinational Finance Journal 2:245-267.

Keim, D. B., and Stambaugh, R. F. 1986. Predicting returns in the stock and bond markets. Journal of Financial Economics 17:357-390.

King, M.; Sentana, E.; and Wadhwani, S. 1994. Volatility and links between national stock markets. Econometrica 62:901-933.

Lee, U. 1997. Stock market and macroeconomic policies: New evidence from Pacific basin countries. Multinational Finance Journal 1:273-289.

Lin, W. L. 1992. Alternative estimators for factor GARCH models-A Monte Carlo comparison. Journal of Applied Econometrics 7:259-279.

Lo, A., and MacKinlay, A. C. 1990. Data snooping biases in tests of financial asset pricing models. Review of Financial Studies 3:431-467.

Lo, A., and MacKinlay, A. C. 1995. Maximizing predictability in the stock and bond markets. NBER working paper No. 5027.

Lo, A., and MacKinlay, A. C. 1997. Maximizing predictability in the stock and Bond Markets. Macroeconomic Dynamics 1:102-134.

Merton, R. C., 1980. On estimating the expected returns of the market. Journal of Financial Economics 8:323-361.

Middleton, L., and Satchell, S. 1999. Deriving the APT when the number of factors is unknown. Quantitative Finance Forthcoming.

Ng, V. M.; Engle, R. F.; and Rothschild, M. 1992ltextbf\{.\} A multi dynamic 
factor model for stock returns. Journal of Econometrics52:245-266.

Poon, S., and Taylor, S. J. 1991. Macroeconomic factors and the U.K. stock market. Journal of Business Finance and Accounting 18:619-636.

Sentana, E., 1995. Quadratic ARCH Models. Review of economic studies. 62:639-661.

Sentana, E., 2002. Factor representing portfolios in large asset markets. Journal of Econometrics Forthcoming.

Sentana, E., and Fiorentini, G. 2001. Identification, estimation and testing of conditionally heteroskedastic factor models. Journal of Econometrics 102:143-164.

Sentana, E., and Wadhwani, S. 1991. Semi-parametric estimation and the predictability of stock market returns: Some lessons from Japan. Review of Economic Studies 58:547-563.

Shiller, R. 1984. Stock prices and social dynamics. Brooking Papers on Economic Activity 2:457-498.

Stambaugh, R. F. 1999. Predictive Regressions. Journal of Financial Economics 54:375-421.

Wong, W. K.; Chew, B. K.; and Sikorski, D. 2001. Can the forecasts generated from E/P ratio and bond yield be used to beat stock markets? Multinational Finance Journal 5:59-86.

Wooldridge, J. M. 1990. A unified approach to robust, regression-based specification tests. Econometric Theory 6:17-43. 